\title{
Las prácticas simuladas en la formación de enfermeros
}

\author{
Mariana-Ornique María ${ }^{1}$, Gabriela-Felippa Silvia ${ }^{1}$
}

\section{INTRODUCCIÓN}

Los orígenes de las prácticas simuladas se remontan al ejército prusiano del siglo XIX. En aquella época se utilizó la simulación para evaluar el comportamiento de cada candidato ante una situación determinada, sustituyendo la evaluación oral. Esta práctica fue utilizada en el entrenamiento de los militares británicos y en el ejército americano, con el estallido de la segunda guerra mundial (1).

En términos de dispositivo de formación de profesionales, las prácticas simuladas tienen como objetivo que los alumnos aprendan mediante la participación y experimentación de una situación, pero sin los riesgos que podría implicar dicha situación «real». La práctica consiste en replicar una situación donde los estudiantes participen de una experiencia de aprendizaje y de formación.

Según Saunders y Powell «La simulación es definida como una representación parcial de la realidad, seleccionando características cruciales y replicándolas dentro de un ambiente o escenario libre de riesgos, al tiempo que permite a los estudiantes a desarrollar sus estrategias y sus estilos para hacer frente a un desafío particular» (2). En algunas circunstancias, la preparación para la actuación profesional requiere obligadamente de la construcción de experiencias simuladas ante las consecuencias que podría acarrear una práctica inexperta en una situación real (3). Casos paradigmáticos son la simulación de vuelo y la simulación en medicina donde está en riesgo la vida.

Dado que se intenta una similitud entre la simulación y la situación real, la aplicación de lo que se aprende es más directa y concreta $\mathrm{y}$, a la vez, permite tomar conciencia de las características, particularidades y presiones de la profesión, especialmente atendiendo debilidades, fortalezas y aspectos por mejorar.

Existen dos prácticas similares entre sí: la simulación y el simulacro. Si bien ambas tienen como finalidad acercar lo que ocurre en el contexto real y son actividades grupales, presentan algunas diferencias. Mientras que la simulación es un ejercicio que se desarrolla en un escenario ambientado, el simulacro se realiza en un escenario lo más similar posible a la realidad.

La simulación es una actuación que colabora en realizar un acercamiento sobre la experiencia y, de este modo, permite que los estudiantes actúen, asuman tareas, cumplan un rol que implica la toma de decisiones (4). De este modo, someten a prueba sus conocimientos, adquieren experiencia, destreza en el desempeño de la tarea, comprenden la complejidad del escenario y generan relaciones entre la teoría y la práctica. También suelen propo-

Tabla 1. Diferencias entre simulación y simulacro

\begin{tabular}{ll}
\hline \multicolumn{1}{c}{ Simulación } & \multicolumn{1}{c}{ Simulacro } \\
\hline Normalmente se realiza en un salón & Se realiza en el terreno; es decir, donde podría darse el desastre \\
Resulta menos costosa & Requiere mayores recursos humanos y materiales. \\
Manejo de información & Ejecución de operaciones \\
Fácil de controlar por el facilitador durante su desarrollo & El control es más complejo \\
Se evalúan actitudes personales y conocimientos & Se evalúa la ejecución de los planes operativos de respuesta \\
\hline
\end{tabular}

Fuente: Federación Internacional de Sociedades de la Cruz Roja y de la Media Luna Roja (FICR). 2008. Guía práctica para la realización de simulaciones y simulacros. San Salvador, El Salvador. Pág. 19. 
nerse instancias donde analizan y debaten acerca de las actividades realizadas, revisan hipótesis de trabajo, evalúan las rutinas y las destrezas puestas en juego. Por su parte, el simulacro es un ejercicio de ejecución de acciones, previamente planeadas, que representan situaciones de desastre semejantes a la realidad y que a través de la movilización de recursos, permite evaluar la capacidad de respuesta ante emergencia o desastre.

La palabra simulación proviene del latín simulat-o, que es la acción de simular (5). Este verbo alude a representar algo imitando o fingiendo lo que no es. La simulación es, entonces, la acción y el efecto de simular. El diccionario de María Moliner proporciona varias acepciones, una de ellas es «hacer parecer que existe u ocurre una cosa que no existe u ocurre» (6).

Las connotaciones de la palabra dan cuenta de una acción «en escenario similar al real» de personas que se encuentran desempeñando la profesión y participan de un «como si» fueran profesionales. Casi todos los aportes sobre las simulaciones como dispositivo de formación de profesionales plantean que las simulaciones acercan al contexto real sin los riesgos de dichas situaciones. Ahora bien, si bien se participa de un «como si», las escenas construyen una realidad. Esas escenas ponen y exponen a quienes participan en ellas a la asunción del rol profesional que poco tiene de ficcional.

En la simulación se construye una escena con visos de realidad que involucra a todos los participantes de la situación. La potencia de esa construcción se da tanto para los que llevan adelante la situación (asumen un rol profesional) como para los que participan de ella observando.

\section{EL MARCO DE LAS PRÁCTICAS SIMULADAS}

La simulación, tal como ha sido mencionado previamente, ha sido planteada como un procedimiento de entrenamiento para el desarrollo de habilidades y destrezas. Las simulaciones parten de la premisa «lo que oigo lo sé, lo que veo lo entiendo y lo que hago lo recuerdo». Persiguen el objetivo de «aprender haciendo».

La experiencia y las reflexiones que se relatan a continuación se inscriben dentro del paradigma de la formación docente de profesionales. Desde allí se valora la experiencia profesional y la reflexión sobre la práctica docente, en tanto espacio de producción de saberes y el reconocimiento a los profesores como profesionales que tienen teorías implícitas y experiencias que pueden contribuir a la constitución de una base sistematizada de cono- cimientos sobre la enseñanza (7).

Las prácticas simuladas constituyen uno de los dispositivos centrados en interacciones que privilegian el intercambio y la confrontación entre pares. Son propuestas que favorecen el ejercicio de la observación, el desarrollo de competencias comunicativas, la formulación y recepción de retroalimentaciones, la integración de conocimientos de diferentes disciplinas y la articulación entre teoría y práctica.

Se trata de un dispositivo que puede propiciar una práctica reflexiva, prestando especial atención a las decisiones que los enfermeros toman en el proceso de diseño de las acciones, la ejecución y evaluación de sus propias prácticas.

Un aspecto de relevancia en las prácticas simuladas es que estimulan y motivan a los involucrados, ya que se trata de una situación vivencial donde pasan a ocupar un rol profesional. Se ponen en juego habilidades y competencias. Por el tipo de actividad propuesta, los alumnos deben actuar, participar, comprometerse y tomar decisiones. Como sostiene Litwin (2008):

«Se trata de aprender en situaciones de práctica, en tanto reconocemos que si los estudiantes participan efectivamente en la organización y desarrollo de una situación, en la búsqueda de información, experimentando alternativas diferentes de resolución, e involucrándose y asumiendo riesgos, los aprendizajes son más duraderos, impactan en sus conciencias, promueven reflexiones y permiten mejores procesos de autoevaluación. Por otra parte, es posible analizar y distinguir las conductas (e hipotetizar sí podrían haber sido otras), comparar acciones y responsabilidades. En todos los casos, las experiencias de simulación se constituyen en una invitación interesante para el análisis posterior, que se transforma en una nueva propuesta de aprendizaje» (3).

Los aportes de Schön (1987) abren una vía distinta para reconstruir los modos en que los profesionales piensan sus prácticas. De allí que el concepto de practicum reflexivo, es fundamental (8).

«Un practicum es una situación pensada y dispuesta para la tarea de aprender una práctica. En un contexto que se aproxima al mundo de la práctica, los estudiantes aprenden haciendo, aunque su hacer a menudo se quede corto en relación con el trabajo propio del mundo real. Aprender haciéndose cargo de proyectos que simulan y simplifican la práctica, o llevar a cabo, relativamente libre de las presiones, las distracciones y los riesgos que se dan en el 
mundo real al que, no obstante, el practicum hace referencia» (9).

Schön piensa en el practicum reflexivo como un instrumento de mejoramiento continuo del proceso de mediación educativa tutor - aprendiz, que maneja diádicamente la reflexión y la acción (10).

Esta visión implica un «giro» respecto de los orígenes positivistas y conductuales de las simulaciones. Resignificadas, las prácticas simuladas se transforman en un dispositivo mediador para que los futuros profesionales puedan pensar-se, conocer-se, estimar-se, regular-se, en relación con otros sujetos implicados y con una situación histórico-social y culturalmente contextualizada. Resulta interesante recordar el planteo de Schön acerca del desafío en la enseñanza de una práctica profesional (11). El autor expone que, en su etapa de formación, los estudiantes deben aprender hechos y operaciones relevantes para su práctica profesional. Pero esto no es suficiente, los enfermeros deben contemplar, también, las formas de indagación que sirven a los profesionales competentes del cuidado para razonar acerca del mejor camino a seguir en situaciones problemáticas.

A través de una simulación se ponen en situación, en acción y en práctica las tensiones que presenta la situación real, las que luego se articulan con conceptos teóricos. Estas prácticas permiten aprender sobre situaciones complejas, procedimientos y operaciones relevantes para la práctica profesional. Adicionalmente a ello, las situaciones vividas pueden ser analizadas desde múltiples perspectivas.

\section{TIPOS DE SIMULACIONES EN EL CAMPO DE LA ENSEÑANZA DE LA ENFERMERÍA}

Pueden clasificarse cuatro tipos de simulaciones que varían en función del objetivo de formación y de la complejidad de la situación (12-13).

1. Simulaciones realizadas con el apoyo de aparatología: cardiorrespiratorio, multipropósitos, obstétricos, etc. Generalmente incluyen simuladores de modelos anatómicos, (maniquís o simuladores de baja fidelidad). El objetivo de su empleo es minimizar el sufrimiento innecesario al paciente y errores en la realización de las técnicas.

Su potencialidad radica en el desarrollo de habilidades y destrezas, relacionadas con la aplicación de procedimientos y técnicas, la exploración física, etc.
2. Simulaciones centradas en el desarrollo de destrezas audiovisuales: se hace una presentación de audio y videos (de forma aislada o integrados en simuladores de alta fidelidad). El objetivo es mejorar la capacidad de interpretación de datos en el paciente, posibilitando la correcta identificación del problema. Su potencialidad radica en el desarrollo de la capacidad de observación y de habilidades auditivas.

3. Simulaciones basadas en el manejo de problemas de pacientes: descripción de la actuación profesional ante una situación clínica: El objetivo es que los alumnos puedan tomar decisiones con responsabilidad y justificación, reproduciendo las decisiones que un profesional debe adoptar y la conducta a seguir con un paciente. Este tipo de simulación potencia el desarrollo de la capacidad de análisis y el proceso de toma de decisiones.

4. Simulación de escenarios clínicos: ejecución de la actuación profesional en una situación clínica. El estudiante interactúa, en un entorno que simula la realidad, con una serie de elementos, a través de los cuales resolverá un caso clínico. El objetivo es que el alumno pueda poner en práctica competencias integrales en una situación lo más parecida posible a la real.

Estas simulaciones son las más complejas y ricas para la enseñanza. Por lo general, incluyen una instancia en la que el docente hace la descripción del material (con qué va a contar el estudiante o grupo de estudiantes para la realización del procedimiento). Luego se presenta la explicación de la práctica (se explicita en qué consiste, qué procedimientos deben hacer, qué se espera de ellos, qué conocimientos pueden ser empleados). A continuación se hace la exposición del caso (aquí el docente describe el contexto de la práctica (unidad de urgencias, terapia, etc., así como los síntomas del paciente). A partir de allí se hace la práctica propiamente dicha $\mathrm{y}$, por último, el análisis de la misma (el docente da lugar a que los participantes y el observador comenten sus impresiones, sensaciones, dudas y por último es el propio docente quien comenta los logros y puntos por mejorar)

En este caso, los alumnos se pueden encontrar con pacientes humanos simulados (con guiones específicos) o con muñecos.

\section{MOMENTOS DE LA SIMULACIÓN}

Cada uno de los tipos de simulación presentados anteriormente puede ser pensado y caracterizado desde sus distintos momentos: 


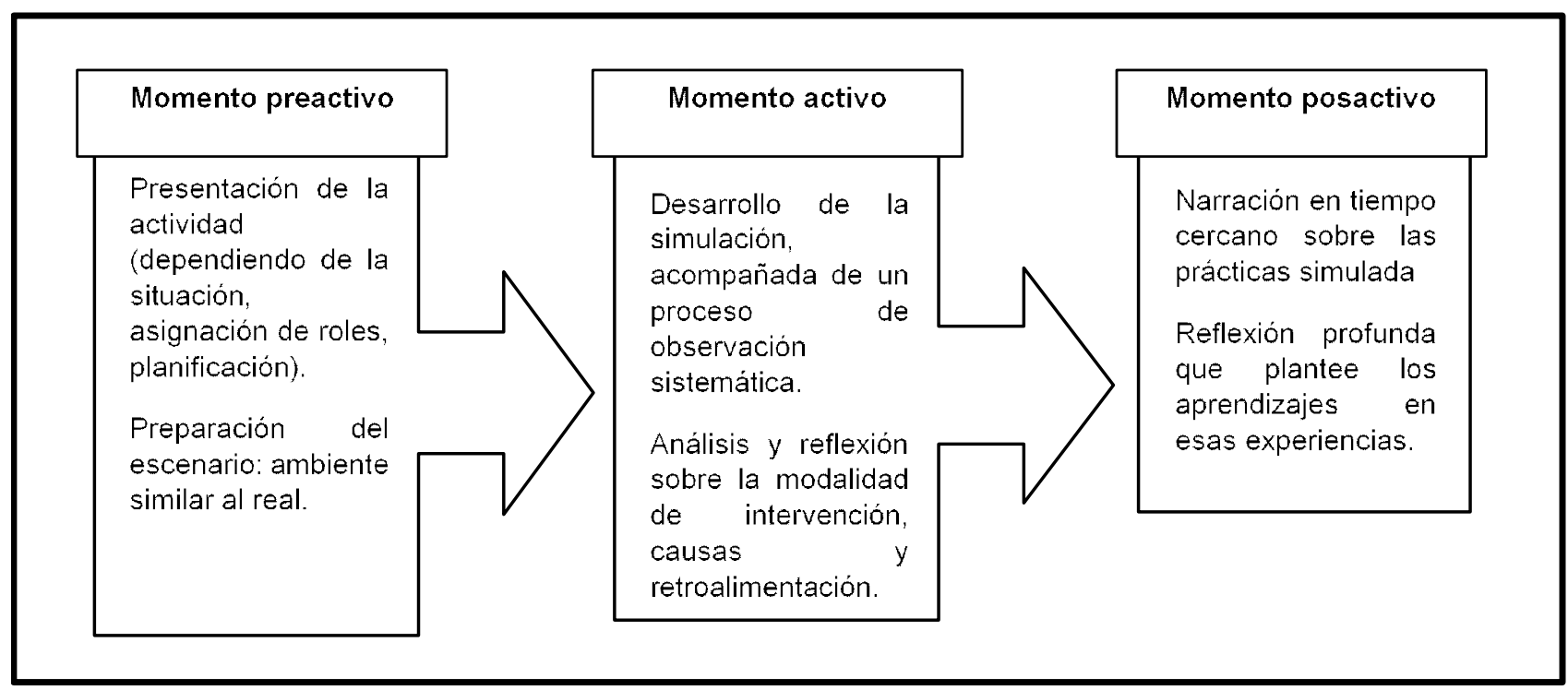

Momento preactivo: en el momento previo a la práctica de simulación es necesario considerar algunas cuestiones en relación al escenario, la situación/problema que se plantea, la distribución de roles y funciones y los tiempos. Este momento requiere de mucha planificación, decisión y organización de recursos por parte del equipo de docentes, no solo para definir el orden y la secuencia de las prácticas sino para determinar la consistencia y coherencia de la propuesta en su conjunto.

La definición de la situación o problema que se intenta generar debe estar vinculada a un contenido y a la adquisición de ciertas competencias. Es clave que los docentes definan qué buscan que aprendan los estudiantes en estas prácticas y definir los objetivos del ejercicio para luego poder evaluarlos. También es preciso explicitar claramente a los alumnos qué contenidos y procedimientos se enseñarán a través de la actividad y qué competencias pondrán en juego.

Se recomienda considerar las situaciones más habituales con las que se puede enfrentar un enfermero, pensar finales diversos para aplicar en distintas simulaciones y cuestiones que vayan complejizándola progresivamente. Es substancial poder organizar las prácticas de simulación en niveles de complejidad. No obstante, en todos los casos la situación debe generar la sensación de realidad que se plantee un problema que resolver.

Otro aspecto que resulta necesario definir es la extensión de la actividad. Esta no es una cuestión menor, dado que la duración generará un efecto psicológico cuanto más crítica y extensa sea la situación propuesta.
En la definición del escenario es necesario considerar el ambiente físico o contexto de trabajo lo más auténtico posible, por ejemplo, efectos tales como olores, situaciones de emergencia, demandas de los distintos actores, sensaciones, ambientes de tensión, confusión e incertidumbre símiles a las situaciones que pueden darse en el ambiente profesional.

Es relevante considerar las proporciones de los espacios y definir los recursos necesarios. Se pueden usar diferentes contextos y promover estrategias de trabajo, generando distintas condiciones de enseñanza y de aprendizaje (14).

Otro punto por considerar es la asignación de roles y funciones, la distribución de tareas que desarrollará cada uno de los participantes, pueden ser grupales o individuales. Es importante que la asignación de tareas sea lo suficientemente general como para que quien participa tenga un importante grado de autonomía para tomar decisiones. De lo contrario, se verá obligado a seguir un guión prefigurado.

Es importante llevar un registro de qué alumnos van desempeñando determinadas funciones para garantizar que cumplan diferentes roles. También, es necesario tener en cuenta que más allá del rol que ocupe cada uno, se aprende de la situación, estando en un lugar u en otro. Esta práctica resulta un aprendizaje tanto para los que están involucrados en la situación como para aquellos que estarán observando y elaborando los respectivos registros. Estos últimos desarrollarán su capacidad de observar pero también de análisis mostrando evidencias, alternativas de 
acción y formulando interrogantes.

Respecto del momento preactivo, sostener la observación sistemática es fundamental para la posterior reflexión sobre las acciones desarrolladas. Esto supone planear quiénes van a observar sistemáticamente y cómo lo van a hacer. Implica en el análisis posterior al desarrollo de las simulaciones que se tomen los registros de observación y se reflexione sobre lo realizado.

Aprender a observar es fundamental, aprender a poner «foco», a construir evidencias, a realizar registros narrativos. Por, ello la definición de observadores en la simulación es tan relevante como la de los que la llevarán adelante simulando el rol profesional.

Momento activo: es el momento de la práctica propiamente dicha. Se despliegan los roles y las acciones tal como se haya planificado. En el momento de la ejecución se recomienda dejar actuar al equipo de estudiantes sin realizar ningún tipo de intervención. Brindar los tiempos necesarios para que puedan interiorizar y pensar sobre la situación. No generar actitudes, comentarios o situaciones que los hagan cambiar de actitud.

En el caso de realizar filmaciones sobre las simulaciones, los estudiantes tendrán la posibilidad de revisar las acciones, visualizarse en cuestiones que tal vez no fueron tan claras para ellos, o no recuerdan.

A continuación, se sugiere especialmente realizar una instancia de reflexión de lo sucedido. Se trata de generar un momento en el que recuperar lo sucedido en la actividad a través de aquellos que observaron y tomaron nota, así como las vivencias de los testimonios de quienes interpretaron roles claves, para que, a partir de allí, se genere un intercambio. Se propone que grupalmente puedan rescatar las vivencias, los sentimientos y sensaciones, revisar conocimientos puestos en juego y extraer conceptos claves que luego puedan ser trabajados en clases teóricas, y así profundizar los vínculos entre la teoría y la práctica así como mirar la práctica desde distintos marcos teóricos.

Es necesario que una vez finalizada la experiencia se analice su desarrollo y la participación de los distintos involucrados, las dificultades que se afrontaron, las soluciones, y las alternativas posibles.

Puede darse un tiempo de trabajo grupal con consignas, con protocolos que permitan analizar acciones, relacionar con materia/s de las asignaturas vinculantes, realizando preguntas que no solo sean cerradas sino abiertas y diver- gentes, por ej. ¿Por qué se tomaron las decisiones que se tomaron?, si hubiera sucedido tal cosa....ंqué decisión hubieran tomado? Estas preguntas deben dar cuenta de demandas cognitivas complejas.

Esto ayudará a construir el diálogo y a la construcción del criterio desde el rol profesional. Preguntas como: ¿por qué?, ¿qué acciones diferentes se podrían haber desarrollado?, ¿qué decisión, cuándo y cómo tomarla? deben servir de guía para la simulación y para el análisis posterior. Dicha propuesta difiere de una práctica guiada porque no solo hace foco en el dominio de la destreza o técnica a implementar sino que se complejiza en el sentido de que la simulación se sitúa en un contexto y pone de relieve la toma de decisión y los criterios a través de los cuales se puede optimizar una intervención.

Puede suceder que el intercambio posterior se realice a través de preguntas que intenten: a. Identificar determinados contenidos, conocimientos o habilidades específicas, más que promover una reflexión desde los cursantes, una autoevaluación de su actuación o la de sus pares; b. Identificar aspectos correctos e incorrectos del procedimiento; c. Analizar alternativas de actuación, ligar los protocolos a la construcción de un «criterio profesional».

En este sentido, es central la tarea del docente para generar un análisis de profundidad y de reflexión a través de preguntas que los induzcan a pensar, comparar y argumentar con sus pares, sin quedarse en lo anecdótico ni en la descripción pura, habilitando conexiones teóricas que probablemente los alumnos solos no logren hacer (15). La potencia de las simulaciones no dependerá solo de su diseño o del ambiente en sí, sino de las competencias y habilidades que los docentes puedan transmitir en el transcurso de toda la actividad, generando instancias de intercambio, revisión y reflexión de lo sucedido.

\section{Sobre la importancia de la retroalimentación en las simulaciones}

Tal como señalan Anijovich y González, el concepto de retroalimentación surgió en el campo de la ingeniería de sistemas, aludiendo a una información que, de algún modo $\mathrm{u}$ otro, tiene impacto en alguna estructura de funcionamiento (16). Según Von Bertalanffy la retroalimentación es un proceso de regulación de los sistemas (16).

En el campo de la enseñanza, la retroalimentación o feedback constituye una herramienta valiosa a través de la cual los docentes podemos ayudar a los alumnos a que regulen su propio proceso de aprendizaje. En ese sentido, habilita una mirada hacia atrás (hacia aquello que ya se 
hizo) para poder mirar hacia el futuro (hacia aquellas acciones que se van a desarrollar en adelante). De este modo, podemos ofrecer información a los alumnos sobre qué y cómo está aprendiendo para que pueda aprender más y mejor.

Ejemplos de feedback pueden ser valoraciones positivas en las que se indique aquello que fue realizado adecuadamente así como sugerencias de mejora en la que se señale aquellos puntos o aspectos a modificar acompañado de un cómo. Asimismo, se puede acompañar con buenos ejemplos o con contraejemplos, con preguntas, reflexiones, etc.

Para que verdaderamente la retroalimentación constituya una contribución a los aprendizajes de los alumnos, sostienen los autores anteriormente mencionados, es necesario acotar el área sobre la cual se va a dar devolución. Por ejemplo, si se está enseñando a elaborar registros narrativos es recomendable recortar uno o dos focos sobre los cuales dar retroalimentación. En este caso podría ser la separación entre hechos observados e impresiones personales o las sugerencias para quien está ejecutando la acción observada. Cuando damos devolución sobre muchas áreas a la vez, perdemos profundidad y oportunidades para propiciar aprendizajes más profundos.

Asimismo, es importante que la retroalimentación se efectúe lo más cerca posible de la tarea realizada. De ese modo, tendrá más sentido para el alumno y podrá retomar en próximas situaciones aquello señalado por el docente o por sus propios pares.

Luego de las simulaciones, el feedback brindado por el docente debería empezar por quienes simularon, para lo cual es importante conceder el tiempo suficiente para la respuesta. El necesario revisar la conducta, nunca a la persona, describir, interpretar, no juzgar. Debe proporcionarse de manera respetuosa y con interés. En el feedback es importante centrarse en las fortalezas y en los puntos a resolver y focalizar lo que se devuelve en algunos aspectos por simulación. No se puede devolver todo en todas las devoluciones. Hay que elegir que foco de devolución se realiza en cada simulación.

Respecto de la intervención posterior a la simulación, se sugiere al docente no ofrecer una retroalimentación negativa inmediatamente a los practicantes. Esta es una forma de intervención posible que puede ser necesaria, pero también es importante generar situaciones en las que el docente oriente o promueva momentos dentro de la práctica para reflexionar colectivamente, intercambiar opinio- nes y que pueda surgir una devolución constructiva por parte del grupo.

En cuanto al tipo de comunicación, se propone una salida de lo «radial» de preguntas planteadas por el docente y respuestas breves del alumno, centrándose en un conocimiento convergente, a través de preguntas convergentes (de única respuesta) y de demanda cognitiva simple (respuestas breves de evocación de conocimientos/ procedimientos). Es necesario generar otro tipo de instancia mediatizada que capitalice las observaciones realizadas por los alumnos.

Cuando las prácticas se filman, sería conveniente además del intercambio post simulación, en algunas ocasiones, pasar la filmación de la simulación al grupo que participó de ella para «verse» nuevamente, analizarse con distancia, estableciendo nuevas conjeturas, profundizando ideas ya discutidas.

Para que las prácticas simuladas contribuyan a la reflexión y no solo al entrenamiento, es imprescindible dedicar tiempo a la reflexión, orientarla, definir focos y valorar los aportes de los alumnos en el momento de devolución. Para ello, el docente mismo puede ofrecer retroalimentaciones sobre las reflexiones de los alumnos, por ejemplo, haciendo señalamientos como: «Es interesante esa idea porque...», o hacer preguntas que permitan la reflexión como, ¿qué es lo que está marcando esa sugerencia?, ¿qué podemos pensar sobre la reflexión de X?

Momento posactivo: finalmente, en el tercer momento se pueden distinguir por lo menos dos instancias de reflexión. La primera, en la cual el alumno/a redacta en un tiempo cercano a la experiencia «la escena simulada». Para ello recibe la siguiente consigna:

«Luego de realizar la simulación tómense un tiempo para considerar algún suceso inesperado. Es condición imprescindible que esté ligado a algo que les haya sucedido en esa simulación. Reflexiones sobre lo que se quedó pensando... Hay que recordar la situación, narrarla, formular hipótesis acerca de lo ocurrido. Como puedan... En un ejercicio de reflexión. Y entregarla la semana posterior a la fecha de la simulación. En papel».

Este es el momento en el que el alumno realiza un trabajo de construcción de significados sobre su práctica simulada, a partir de un diálogo que debe construir, en el que intenta primero describir lo que ocurrió para, posteriormente, tratar de comprenderlo, utilizando además de las 
opiniones personales basadas en creencias e ideas previas, conocimientos provenientes del campo teórico de la salud y la devolución que le hicieron sus compañeros y docentes.

Luego de haber realizado varias simulaciones, se solicita una reflexión profunda que plantee los aprendizajes en esas experiencias.

Respecto del análisis de los registros que toman los alumnos a lo largo de las simulaciones, una alternativa es que un alumno elija un registro exhaustivo desde el cual se realice un análisis sobre lo actuado y le permita construir una actitud crítica y reflexiva sobre la intervención. Para esta actividad se les puede pedir que lleven registros previos «pasados en limpio».Con los registros narrativos es importante que primero se describa qué se hizo para luego abordar: las fortalezas de la situación y, posteriormente, señalar aspectos a revisar / progresar y luego analizar las acciones. Los registros dan las evidencias para el aná-

Tabla 2. Tabla de registro de lo aprendido en las simulaciones

Evidencias $\quad$ Sugerencias

\section{Fortalezas}

Aspectos a

revisar

\section{ACERCA DE LAS POTENCIALIDADES, LOS RIESGOS Y LÍMITES DE LAS SIMULACIONES}

Se trata de promover centralmente la reflexión sobre las prácticas. De esta manera, «las prácticas simuladas se transforman en un dispositivo mediador para que los futuros profesionales puedan pensar-se, conocer-se, estimarse, en relación con otros sujetos implicados y con una situación histórico-social y culturalmente contextualizada» (18). Permiten trabajar sobre las emociones que se ponen en juego, aprender de situaciones problemáticas, analizar, comprobar, constatar nuevas categorías.

El uso de este dispositivo da una posible respuesta al desafío de aprender una práctica profesional ${ }^{1}$. Esto significa aprender habilidades, destrezas, y modos de proceder, ejercitar modos y estrategias de acción. De esta manera, va a construir criterios para identificar la mejor manera de proceder en situaciones complejas e internalizar distintas formas de indagación sobre lo sucedido para que se adaptadas de manera reflexiva en la práctica profesional. lisis y presencia o ausencia del pensamiento reflexivo por parte del estudiante (17).

En este momento es importante destacar las cuestiones de mayor fortaleza, por ejemplo, focalizarse en el trabajo en equipo. Debe ayudarse al estudiante a que identifique y registre evidencias de lo aprendido y ejemplos concretos en la simulación observada, así como dar sugerencias sobre ello. Puede generarse recursos didácticos que ayuden al estudiante a sistematizar su reflexión y a identificar que puede generar nuevas hipótesis de lo observado. Finalmente, puede motivarse al estudiante a que comparta con sus compañeros su producción.

El siguiente esquema podría ayudar a sistematizar su reflexión.

Tabla 3. Tabla de registro de nuevas preguntas de indagación e hipótesis

\begin{tabular}{|l|l|}
\hline A partir de la simulación me & a) \\
formulo las siguientes & b) \\
preguntas: & c) \\
\hline Establezco una conjetura o & \\
hipótesis de trabajo acerca de & \\
la perspectiva de abordaje de & \\
la enfermería & \\
\hline Establezco una conjetura o & \\
hipótesis de trabajo acerca & \\
del.... & \\
\hline
\end{tabular}

Más allá de las potencialidades de las prácticas simuladas se presentan algunos riesgos y limitaciones que son necesarias tener en cuenta.

La génesis de las simulaciones en las formaciones profesionales está tan indisolublemente ligada al desarrollo de habilidades, al entrenar técnicas, que por ello resulta un cambio paradigmático muy grande el giro a la reflexión. Queda todavía mucho recorrido por realizar para que la reflexión sea el marco de las simulaciones y no solo el «entrenarse».

Las simulaciones se desarrollan en un contexto artificial y resulta importante que los estudiantes sean conscientes de esta limitación, que debe ser explicitada y trasparentada para evitar una visión sobresimplificada, fragmentada y superficial, de las situaciones reales profesionales.

Es necesario comprender, también, que actuar bien en la experiencia construida para el aprendizaje no es el reaseguro de una buena actuación en una situación análo- 
ga. Se trata, simplemente, de la adquisición de la experiencia y, por lo tanto, de práctica y no de una actividad predictiva.

«La presente publicación ha sido elaborada con la asistencia de la Unión Europea, en el marco del proyecto Alfa Funda Enfermería (Contrato CRIS 279-078). El contenido es de responsabilidad exclusiva de los autores y, en ningún caso, debe considerarse que refleja los puntos de vista de la Unión Europea».

\section{REFERENCIAS BIBLIOGRÁFICAS}

1. Andreu AMA, García CM, Mollar GM.La simulación y juego en la enseñanza-aprendizaje de lengua extranjera. 2005. 11(55): 34-9.

2. Saunders D, Powwell T. Developing a European media simulation through new information and communication technologies: The TENSAL Project. En: Rolfe J, Saunders D, Powell T, editores.Simulations and Games for Emergency and Crisis Management. Londres: BiddlesLtd, Guildford and King's Lynn; 1998. p.75-86.

3. Litwin E. El oficio de enseñar. Condiciones y contextos. Buenos Aires: Paidós; 2008.

4. Amaya AA. Simulación clínica: «aproximación pedagógica de la simulación clínica». Univ. Méd. Bogotá (Colombia). 2010.51 (2): 204-211.

5. Aguirre DGL. Postura experiencial de los docentes que utilizan la simulación clínica como estrategia didáctica en la carrera de medicina [tesis]. Bogotá: Universidad Nacional de Colombia; 2012.

6. Moliner M. Diccionario. Madrid: Gredos; 2000.

7. Anijovich R, Cappelletti G, Mora S. Sabelli MJ. Transitar la formación pedagógica. Dispositivos y estrategias. Buenos Aires: Paidós; 2009.

8. Schön DA. Educating the Reflective Practitioner: Toward a New Design for Teaching and Learning in the Professions. Memphis: Jossey Bass Inc.; 1987.

9. Anijovich R, Cappelletti G. LA enseñanza de las prácticas en la formación profesional. I Jornadas Internacionales «Problemáticas en torno a la enseñanza en la educación superior. Diálogo Abierto entre la didáctica general y las didácticas específicas. Ago 2013. Córdoba: Universidad de Villa María. p. $1-11$.

10. LoughranJJ. Effective Reflective Practice In Search of Meaning in Learning about Teaching. Journal of TeacherEducationJanuary. 2002. 53(1): 33-43
11. Schön DA. El Profesional reûexivo. Buenos Aires: Paidós; 1998.

12. Quesada SA, Burón MFJ, Castellanos OA, Del Moral VMI, González FC, Olalla AJJ, Rabanal LJM, Rodríguez BJC, Teja BJL. Formación en la asistencia al paciente crítico y politraumatizado: papel de la simulación clínica. Medicina Intensiva. 2007. 31:(4): 187-193.

13. Gómez BLM. Entrenamiento basado en la simulación, una herramienta de enseñanza y aprendizaje. Rev. Col. Anest. 2004. 32:201-8.

14. Chaiklin S, Lave J. Estudiar las prácticas: perspectivas sobre actividad y contexto. Madrid: Amorrortu Editores. 2001.

15. Serrano CJA. Reseña de «Cómo pensamos. Nueva exposición de la relación entre pensamiento reflexivo y proceso educativo» de John Dewey. Revista Intercontinental de Psicología y Educación. 2005. 7(2):154-62.

16. Anijovich R, Gonzáles C. Evaluar para aprender Conceptos e instrumentos. Buenos Aire: Aique educación. 2013.

17. Chirema K. The use of reflective journals in the promotion of reflection and learning in postregistration nursing students. Nurse Educ Today. 2007;27(3):192-202.

18. Cappelletti G, Ornique M, Sabelli MJ, Sigal C. Las prácticas simuladas en la formación de docentes. IN: Ministerio de Educación, Instituto Nacional de Formación Docente, OEI-IDIE, Xunta de Galicia, Facultad de Derecho de la Universidad de Buenos Aires, Universidad Nacional de General Sarmiento, Universidad de Sevilla, editores. II Congreso Internacional sobre profesorado principiante e inserción profesional a la docencia. El acompañamiento a los docentes noveles: prácticas y concepciones; 24-26 Feb. 2010; Buenos Aires, Argentina; 2010. p. 1-11.

\section{Correspondencia}

María Mariana Ornique

XXXXX

Correo electrónico: XXX@XXX

Forma de citar este artículo: Mariana-Ornique M, GabrielaFelippa S. Las prácticas simuladas en la formación de enfermeros. Rev enferm Herediana. 2013;6(2):115-122. 